\title{
Comportement d'une bulle de gaz piégée dans un liquide soumise à une onde de compression
}

\author{
par C. Bourgeois, N. Djebaili-Chaumeix, A. Quilgars et C. Paillard \\ Laboratoire de Combustion et Systèmes Réactifs, Université d'Orléans
}

\section{I INTRODUCTION}

L'étude du comportement d'une bulle de gaz piégée dans un liquide, soumise à une onde de compression doit conduire à la maîtrise des risques d'explosion des systèmes à hydrazine (moteurs fusée). Ces risques sont engendrés par la présence d'inclusions gazeuses dans l'hydrazine liquide soumises à un coup de bélier. Plus généralement, ce travail apporte des informations nécessaires à la compréhension du mécanisme de détonation d'explosif initié par points chauds. Pour ce faire, nous avons caractérisé la compression de bulles de gaz combustible (1\% $\left.\mathrm{C}_{2} \mathrm{H}_{4}-3 \% \mathrm{O}_{2}-96 \% \mathrm{Ar}\right)$ et inerte $\left(2 \% \mathrm{C}_{2} \mathrm{H}_{4}-98 \% \mathrm{Ar}\right)$ de volume initial compris entre 100 et $1000 \mathrm{~mm}^{3}$, déterminé les conditions d'inflammation des bulles de gaz combustibles et vérifié l'hypothèse d'une compression adiabatique. Par ailleurs, les coefficients isentropiques, en fonction de la température, étant très voisins pour les deux mélanges gazeux combustible et inerte, on peut procéder par comparaison entre une bulle combustible et son analogue inerte, pour déterminer dans les observations faites ce qui est dû au phénomène de compression et ce qui est dû à la réactivité de la bulle.

Peu de travaux concernant la compression d'une bulle réactive isolée au contact d'une paroi apparaissent dans la littérature.
Hasegawa et Fujiwara $[1,2]$ ont étudié la compression de bulles inertes ou réactives $\left(30 \%\left(2 \mathrm{H}_{2}+\mathrm{O}_{2}\right)-70 \% \mathrm{Ar}\right)$, de diamètre initial compris entre 9 et $12 \mathrm{~mm}$, piégées en général dans la glycérine. Quelques essais ont été réalisés dans l'eau montrant ainsi l'influence de la nature du liquide. Les travaux des Japonais sont intéressants car le dispositif expérimental, les niveaux de pression et les dimensions des bulles se rapprochent de ceux utilisés dans cette étude.

Ils ont pu mettre en évidence, pour le cas de bulles inertes ou en l'absence d'inflammation, des cycles de compression dans lesquels la bulle est soumise à des compressions et des expansions successives. Ils ont montré que la compression d'une bulle s'accompagne d'émission d'onde de pression qui s'atténue au cours de leur propagation. Leurs deux publications sont en désaccord sur les conditions d'inflammation d'une bulle réactive de taille comprise entre 9 et $12 \mathrm{~mm}$ sans qu'ils en donnent l'explication.

D'autres auteurs, tels que Fujikawa et Akamatsu [3,4] ont travaillé sur des bulles d'un millimètre de diamètre à différentes distances d'une paroi. Ils ont ainsi montré l'influence de cette dernière sur la symétrie de la compression. Parallèlement aux études expérimentales, des modèles de compression de bulles ont été développés. Le plus complet et performant est celui construit par Mazel et Col. [5].

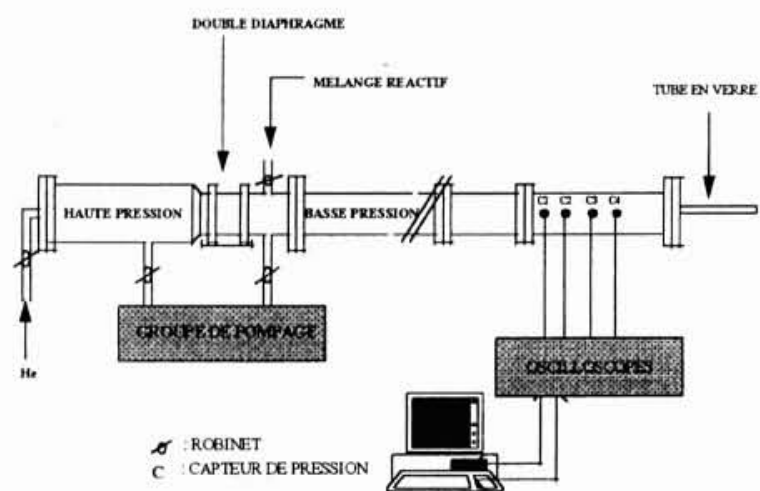

Tube à choc et son instrumentation

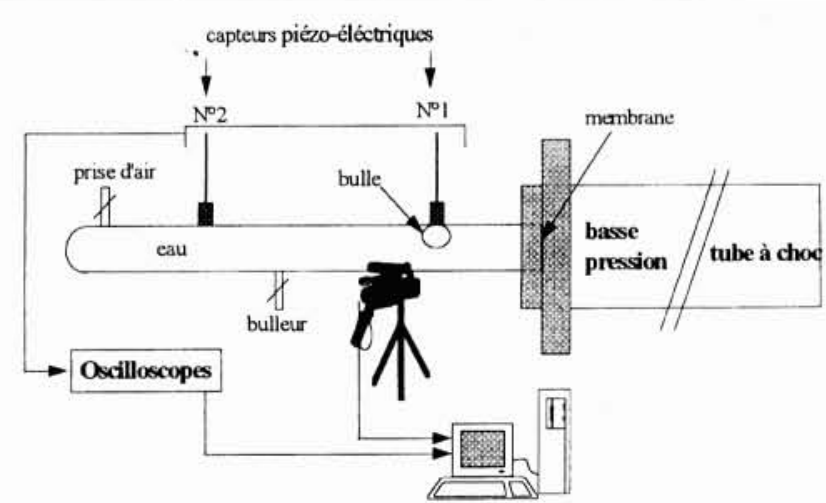

Tube en verre et son instrumentation

1. Dispositif expérimental constitué du tube à choc couplé à un tube en verre 


\section{II — DISPOSITIF EXPÉRIMENTAL}

Le dispositif expérimental utilisé dans cette étude est un tube à choc au bout duquel a été fixé un petit tube en verre rempli d'eau et contenant la bulle à étudier (Fig.1). L'étanchéité entre les deux tubes est réalisée par une membrane en terphane de $50 \mu \mathrm{m}$ d'épaisseur.

Le tube à choc, de $4 \mathrm{~m}$ de long et de $50 \mathrm{~cm}$ de diamètre interne, est constitué d'une partie haute pression remplie par de l'hélium à 1,3 MPa et séparée par un double diaphragme de la partie basse pression contenant de l'air à la pression atmosphérique. Le tube en verre placé à l'extrémité de la partie basse pression est long de $180 \mathrm{~mm}$ et a un diamètre interne de $11 \mathrm{~mm}$. La bulle est, en général, toujours placée sous la premier capteur de pression piézo-électrique à environ $90 \mathrm{~mm}$ de l'extrémité du tube à choc. La dépressurisation rapide du compartiment à double diaphragme conduit à la rupture successive des deux membranes, qui le constituent, générant ainsi la formation d'une onde de choc dans la partie basse pression du tube à choc. A l'extrémité du tube à choc, une partie de l'onde de choc incidente est réfléchie et génère une pression $\mathrm{P} 5$, dite pression génératrice de choc, de 2,1 $\mathrm{MPa}$ environ et constante durant toute la compression de la bulle. Une onde de compression est transmise au liquide contenu dans le tube en verre et sert à la formation de l'onde de compression responsable de la compression de la bulle.

Quatre capteurs de pression piézo-électriques situés à l'extrémité du tube à choc affleurant à la paroi permettent de détecter le passage de l'onde de choc incidente et de mesurer sa célérité. A partir de cette mesure et des différentes équations de conservation, on détermine la pression génératrice de choc, P5. La pression au voisinage de la bulle est enregistrée par deux capteurs de pression piézo-électriques situés respectivement au dessus de la bulle et à $75 \mathrm{~mm}$ en aval. Une caméra rapide enregistre à une fréquence de 27000 images. $\mathrm{s}^{-1}$ la déformation de la bulle.

\section{III — RÉSULTATS}

Dans notre configuration expérimentale, la bulle est collée à la paroi et prend une forme allongée que l'on caractérise par sa longueur D. On constate sur la figure 2 une déformation asymétrique de la bulle lors de sa compression. L'extrémité du ménisque opposé au choc reste fixe par rapport à la paroi prati-

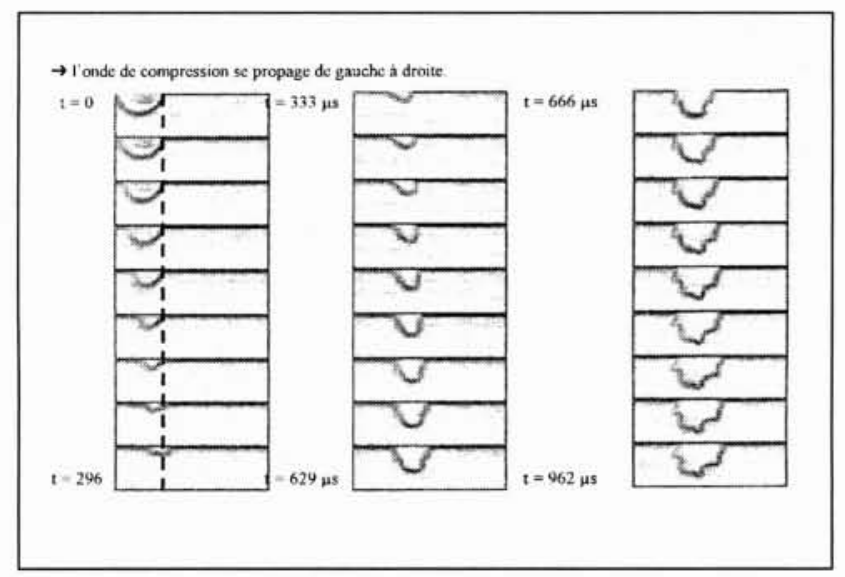

2. Images de la compression d'une bulle de taille initiale $8 \mathrm{~mm}$ et constituée de $2 \% \mathrm{C}_{2} \mathrm{H}_{4}$ - $98 \%$ Ar. L'intervalle de temps entre chaque image est de $37 \mu$ s. La pression génératrice du choc est de $2,7 \mathrm{MPa}$.

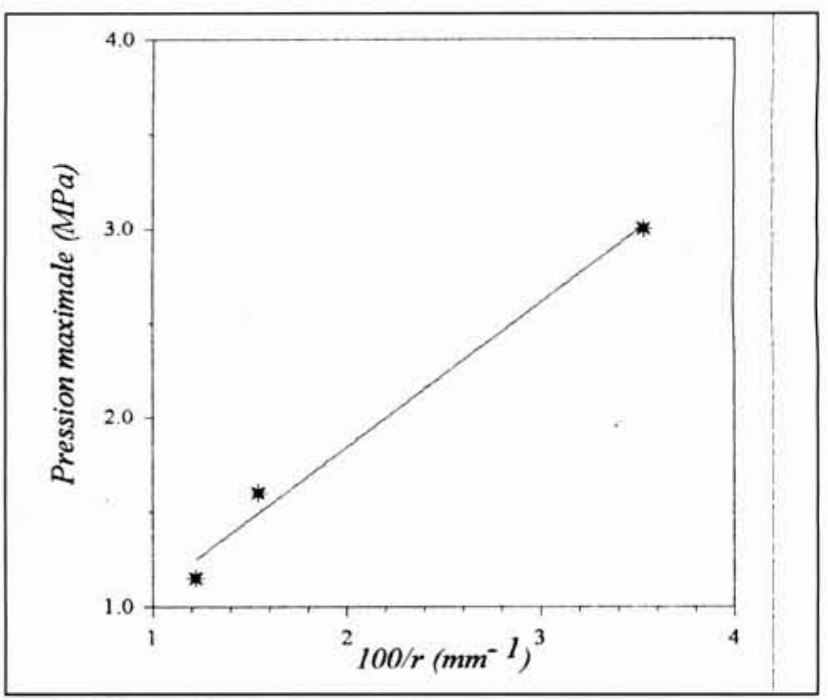

3. Evolution, au cours de la compression d'une bulle, du maximum de pression enregistré dans le liquide en fonction de l'inverse de la distance séparant le premier capteur des trois autres. Bulle de taille initiale de $8 \mathrm{~mm}$ constituée de $1 \%$ $\mathrm{C}_{2} \mathrm{H}_{4}-3 \% \mathrm{O}_{2}-96 \%$ Ar. Pression génératrice du choc de $1,7 \mathrm{MPa}$.

quement jusqu'à la fin de la phase de compression. L'interface gaz-liquide conserve son intégrité uniquement pendant la compression. En effet, on voit très vite apparaître des instabilités de Rayleigh-Taylor à cette interface pendant la phase de l'expansion. Dans ces conditions, on n'observe pas, contrairement à ce que prévoit la modélisation de Mazel et Col. [5], plusieurs cycles complets de compression.

La compression de la bulle donne elle-même naissance à des ondes de pression (fig.3) qui s'atténuent en fonction de l'inverse de la distance séparant le point de mesure et le centre de la bulle (1er capteur). Ces ondes de pression peuvent être apparentées à des ondes hémisphériques.

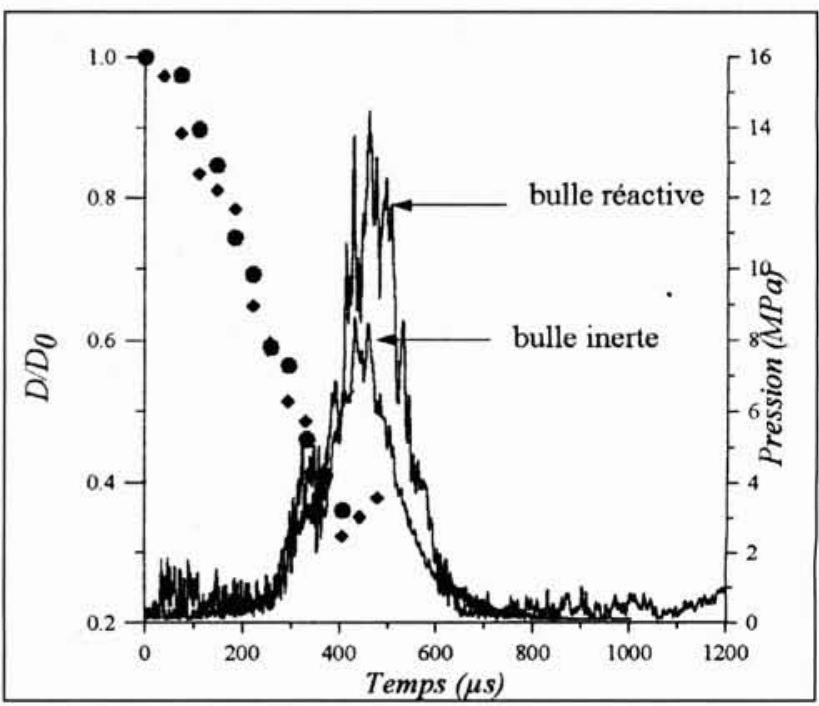

4. Evolution du rapport $D / D 0$, de la pression au voisinage de la bulle ( $1^{\text {er }}$ capteur). Pression génératrice du choc : 2,1 MPa. $\checkmark$ : cas d'une bulle inerte ( $2 \% \mathrm{C}_{2} \mathrm{H}_{4}-98 \%$ Ar).

- : cas d'une bulle réactive (1 \% $\mathrm{C}_{2} \mathrm{H}_{4}-3 \% \mathrm{O2}$ - $96 \%$ Ar). Longueur initiale de la bulle $: 11,5 \mathrm{~mm}$. 


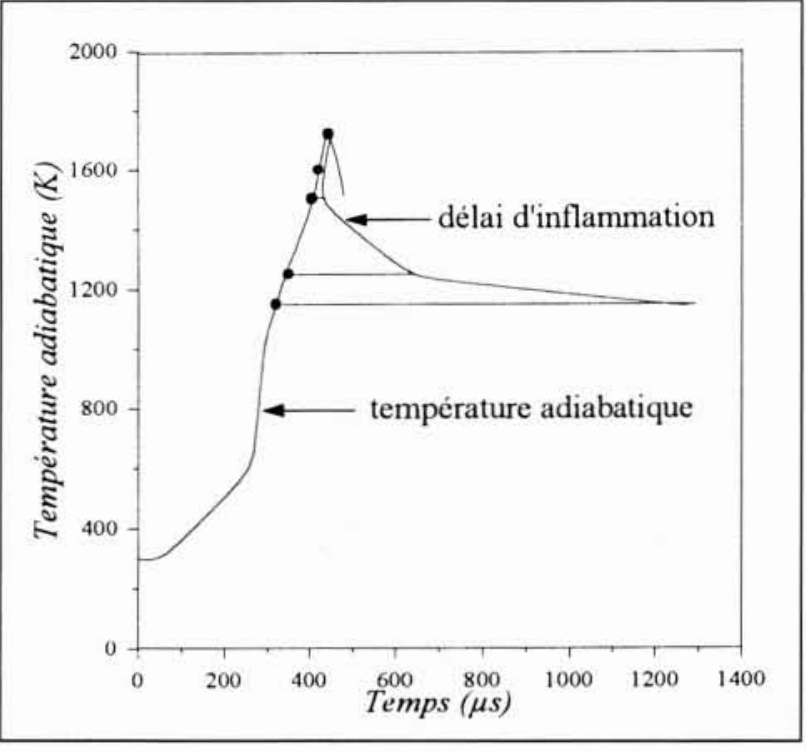

5. Profils de la température adiabatique et du délai d'inflammation correspondant pour une bulle de longueur initiale de $11,5 \mathrm{~mm}$. - température adiabatique ; $\rightarrow$ : délai d'inflammation.

L'inflammation d'une bulle de gaz combustible est principalement détectée par la différence de pression existant entre les cas combustible et inerte (fig.4). Cette différence de pression peut parfois s'accompagner d'une déformation différente de la bulle réactive dans sa phase d'expansion. Pour des bulles de gaz combustible et inerte de longueur initiale comprise entre 8,5 et $16,5 \mathrm{~mm}$, on a respectivement enregistré des pics de pression variant entre 20 et $2,7 \mathrm{MPa}$ et entre 16 et $2,7 \mathrm{MPa}$. L'inflammation n'est plus observée pour des bulles de taille supérieure à $16,5 \mathrm{~mm}$. On a pu constater une variation linéaire du temps de compression avec la taille de la bulle. Ces temps sont compris entre 370 et $780 \mu$ s.

\section{IV — DISCUSSION}

A partir des profils de pression obtenus expérimentalement et du calcul des délais d'inflammation des mélanges $\left(1 \% \mathrm{C}_{2} \mathrm{H}_{4}\right.$ $3 \% \mathrm{O}_{2}-96 \% \mathrm{Ar}$ ), l'hypothèse d'une compression adiabatique est raisonnable dans les conditions de cette étude durant le premier cycle de compression. En effet, en appliquant, pour une taille de bulle donnée, le modèle adiabatique au profil de pression, on obtient le profil de la température adiabatique dans la bulle et on peut calculer les délais d'inflammation correspondants. La confrontation de ce profil de température avec celui des délais d'inflammation a permis de vérifier que les calculs, faits dans l'hypothèse d'une compression adiabatique, confir- ment l'inflammation et la non inflammation observées expérimentalement. Cette confrontation nous a aussi permis de montrer que l'inflammation d'une bulle de gaz combustible n'est possible que sur la première compression.

\section{$\mathrm{V} \square$ CONCLUSION}

Cette étude sur le comportement d'une bulle de gaz piégée dans l'eau et au contact d'une paroi, permet de montrer que les conditions d'inflammation sont étroitement liées à la taille initiale de la bulle. Pour une longueur initiale supérieure à $16,5 \mathrm{~mm}$, l'inflammation de la bulle n'est plus observée. Les niveaux de pression enregistrés au voisinage de la bulle sont relativement élevés, surtout quand il se produit l'inflammation de la bulle.

L'hypothèse d'une compression adiabatique a été validée à partir du calcul des délais d'inflammation des mélanges (1\% $\mathrm{C}_{2} \mathrm{H}_{4}-3 \% \mathrm{O}_{2}-96 \% \mathrm{Ar}$ ) et du profil de température adiabatique de la bulle pour des volumes initiaux compris entre 100 et $1000 \mathrm{~mm}^{3}$. Enfin, on a montré que si l'inflammation devait se produire, elle ne pouvait être initiée que pendant la première compression.

Ces premiers résultats devraient permettre, par extrapolation aux cas de systèmes à hydrazine, de déterminer le domaine de sécurité de fonctionnement des systèmes à hydrazine.

\section{REMERCIEMENTS}

Cette étude a été réalisée dans le cadre d'une thèse financée par le Centre National d'Etudes Spatial et par Matra Marconi Space France.

\section{RÉFÉRENCES}

[1] T. Hasegawa ET T. Fujwara (1982). - Detonation in oxyhydrogen bubbled liquids. 19th Symp. (Internat.) Combustion, édité par The Combustion Institute, Pittsburgh, $\mathrm{Pa}, 675-683$.

[2] T. Hasegawa ET T. Fuitwara (1984). - Propagation velocity and mechanism of bubble detonation. Progress in Astronautics and Aeronautics : Dynamics of shock waves, explosions and detonations, 94, 1379-1396.

[3] S. Fujikawa Et T. Akamatsu (1978). - Experimental investigation of cavitation bubble collapse by a water shock tube. Bulletin of the JSME, 21, 152, 223-230.

[4] S. FunkaWA ET T. AKamaTSU (1975), - Observation of cavitation bubble collapse produced in a water shock tube. 10th Symp. (Intern) on Shock Tubes and Waves, Kyoto, Japon, édité par G. Kamimoto, 174-183.

[5] P. MAZel, R. SAUReL, J.C. LoRAND et P.B. Butler (1996), - A numerical study of weak shock wave propagating in a reactive bubbly liquid. A paraitre dans Combustion Science and Technology. 